Saberes, II - 2020, ISSN: 1794 -4384 (En línea), 1794 -4384 (Impreso)

\title{
EI Derecho Soporte de la Bioética para Garantizar la Salud en Colombia
}

\author{
The Right Support of Bioethics to Guarantee Health in Colombia \\ ${ }^{a}$ Lina Marcela Jiménez Baccal \\ a linam-jimenezb@unilibre.edu.co, Grupo de Investigación "Sociología e Instituciones Políticas”, Semillero de investigación \\ Postconflicto y Justicia” de la Universidad Libre Sede Cartagena. Cartagena, Colombia.
}

Forma de Citar: L.M. Jiménez - Bacca "El Derecho Soporte de la Bioética para Garantizar la Salud en Colombia", Rev. Saberes, Vol. 13, No. 02 , pp. $13-18,2020$.

Recibido: 24/03/2020 Evaluación:28/05/2020 Aceptado: 30/06/2020 DOI: https://doi.org/10.25213/1794-4384/1302.0001

\section{Resumen}

Al momento de unificar la bioética, la salud y el derecho, se conforma una triada para la vida digna, desde el proceso socio jurídico que conllevaría al respeto de los derechos fundamentales e inalienables de los ciudadanos que accedan a la prestación de servicios de salud en las EPS (como la entidad que aprueba o recha un servicio de salud), IPS y clínicas en Colombia. El propósito principal es analizar cómo es necesaria la intervención jurídica en la práctica ética para garantizar los derechos a la salud, a partir del planteamiento de doctrinas que permitan el abordaje de los lineamientos médicos y jurídicos que orienten de la mejor manera a la toma de decisiones desde los conceptos del derecho, la ética, la vida humana y su relación con la prestación de los servicios asistenciales de la salud en Colombia.

\section{Palabras Clave}

Bioética, Derecho, Salud, Dignidad Humana, Humanización.

\section{Abstract}

At the moment of unify ingbioethics, health and law, a triad is formed for decent life, from the socio-legal process that would lead to respect for the fundamental and inalienable rights of citizens who Access the provisión of health services in EPS (such as theentity that approves orrejects a health service), IPS and clinics in Colombia. The main purpose is to analyze how legal intervention is necessary in ethical practice to guarantee the rights to health, based on the formulation of doctrines that allow the approach to medical and legal guide lines that best guide decision-making. From the concept so flaw, ethics, human life and its relationship with the provisión of health care services in Colombia.

\section{Keyworlds}

Bioethics, law, health, human dignity, humanization, rights.

\section{Introducción}

Con este documento el autor pretende demostrarla importancia de la vinculación del derecho con la bioética frente a la necesidad de proteger los derechos fundamentales que hoy día se están vulnerando por la atención deshumanizada de la salud en Colombia, hasta las complicaciones físicas y mentales de los usuarios y familiares.

Integrando la amplia necesidad del respeto por la dignidad humana, la oportuna atención de la salud del enfermo y la preservación de la vida de los ciudadanos colombianos es aquí donde se busca vincular a la bioética y el derecho para salvaguardar los principios, derechos y valores humanos que permitan una atención

\footnotetext{
${ }^{1}$ Autor para correspondencia: correo electrónico $\underline{\text { linam-jimenezb@unilibre.edu.co }}$ 
digna, desarrollando estrategias o modelos para la atención integral en salud de pacientes y sus familias en las IPS, EPS y Clínicas del país.

\section{Metodología}

A partir de lo anterior, utilizando la hermenéutica como herramienta del análisis en primera instancia se define el concepto de la bioética, posteriormente se establece la relación de la bioética con la salud, en una tercera instancia se describe la calidad de la salud en Colombia, luego la articulación de la bioética con el derecho y finalmente la relación de la dignidad humana con la bioética y el derecho.

\section{Resultados y Discusión}

\section{Bioética}

De acuerdo con Francisco León el reconocimiento de la bioética surge históricamente de la ética médica, centrada en la relación médico paciente. Respecto a ésta última, la bioética supone un intento de conseguir un enfoque secular, interdisciplinario, prospectivo, global y sistemático, de todas las cuestiones éticas que acentúan los aspectos sociales o comunitarios: "la Bioética es el estudio interdisciplinar del conjunto de condiciones que exige una gestión responsable de la vida humana (o de la persona humana) en el marco de los rápidos $\mathrm{y}$ complejos progresos del saber y de las tecnologías biomédicas". (1997., p125)

Partiendo de la importancia de conocer a la bioética como una disciplina específica e importante, que busca proponer respuestas a la sociedad y a las problemáticas, que se presentan en las distintas disciplinas de las ciencias de la vida, siendo esta el eje central en la relación médico - paciente que distingue la decisión de una atención deshumanizada en las instituciones prestadoras de salud en Colombia; donde frecuentemente se observa una alta demanda de inconformidades, observándose una vulneración de derechos fundamentales como lo son: la dignidad humana, la vida y la salud.

Por otro lado, en el mismo sentido Alfonso Mendoza plantea que "la aceptación de la salud como un derecho, con el consiguiente crecimiento de la demanda por la atención de salud; y la introducción de las leyes del mercado, que han llevado a que, no pocas veces, el paciente sea visto más como un objeto de lucro que como una persona doliente y necesitada de ayuda. Todo ello explica en gran medida que, al lado de la admiración que nos produce la constatación de verdaderas proezas médicas, crezca también la insatisfacción de los usuarios de los sistemas de salud, hecho que va de la mano con la declinación de los valores éticos profesionales y el severo enjuiciamiento de la profesión médica por parte de la opinión pública" (2017, p 556).

Es importante resaltar que la humanización ha sido un término de gran debate en lo que va corrido del último siglo, porque permite indagar en el sector salud, el trato y atención brindada al ciudadano. Tenemos claro que el avance tecnológico y científico, ha permitido un desarrollo en las ciencias médicas y atención sanitaria en el país, siendo de gran utilidad para mejora de procedimientos médicos. De cierta forma, se ha llegado a interrogante necesario y es, ¿el por qué se ha dejado a un lado la importancia y el desconocimiento que el eje central de la atención en salud sea la persona.

\section{Relación de la Bioética con la Salud}

Nancy Molina Montoya indica que "es necesario que los profesionales sanitarios brinden una atención que promueva la protección de los derechos y la calidad de vida de los pacientes. Señala la necesidad de fortalecer los principios y valores relacionados con el profesionalismo y otras competencias como la identificación de cuestiones morales, el razonamiento moral, la toma de decisiones, la motivación y la acción moral, entre otras" (2016, p 119). 
Por consiguiente la bioética sobrepasa los límites en el campo medico tratando de unir la moral, la ética médica, el saber científico y el desarrollo de la tecnología para brindar una atención humanizada, convirtiéndose en un acto de responsabilidad social, sin embargo no podemos dejar de un lado la necesidad de incorporar a la bioética con el personal no médico que efectúala prestación del servicio de atención al usuario, de ahí que se convierte en el primer momento de un servicio humanizado que se brinda al ingresar a las instituciones prestadoras de salud.

Por dichas razones en necesario resaltar que el desconocimiento de la bioética y la negligencia por parte del personal médico, y no medico en las instituciones de salud debe promoverse el desarrollo continuo de la bioética para la adquisición de habilidades que permitan afrontar las necesidades clínicas de los pacientes y sus familias, de esta manera, la bioética debe incitar a la aceptación de las características culturales, étnicas, cívicosocial, religioso de todas las personas. (Molina et ál., 2016).

\section{Calidad de la Salud en Colombia.}

Según (Restrepo., 2007), la calidad de la salud en el país según la Ley 100 ha logrado resultados muy positivos, en temas como la cobertura y la recuperación económica de muchas instituciones de salud que han logrado adaptarse a la nueva situación, y ha incorporado en la legislación el elemento de solidaridad como un ejemplo digno de reconocimiento. No obstante, podemos observar de cierta forma que la calidad vista desde los factores tales, como la atención oportuna, acceso a los servicios, autorización de medicamentos y procedimientos por parte de las instituciones prestadoras de salud del país es desastrosa, influenciada mayormente por un monopolio económico, donde se deja a un lado las condiciones de salud de los usuarios, por ello existen graves e inminentes problemas en la atención ya que las instituciones no cuentan con las coberturas totales para la atención y muy poca oportunidad para acceder a los servicios.
La calidad viéndose hoy día como la forma de desarrollo, que aporta rentabilidad y crecimiento en cualquier sector productivo del país, observamos que el sector salud no se queda atrás, se ha acogido a la implementación de estrategias que permiten mejorar la calidad de la atención en salud en el país, crecientemente enfocado con "la reforma de la salud a través de la Ley 100 de 1993 que reconoció la calidad como un aspecto fundamental en la atención; el Decreto 1011 de 2006 por el cual se creó el Sistema Obligatorio de Garantía de Calidad de la Atención de Salud del Sistema General de Seguridad Social en Salud, que está constituido por el Sistema Único de Habilitación, la Auditoría para el Mejoramiento de la Calidad de la Atención de Salud, el Sistema Único de Acreditación y el Sistema de Información para la Calidad." (Yépez et ál., 2018).

Por otra parte, tenemos a la Entidad Super Salud como la encargada de vigilar y proteger los derechos de los usuarios afiliados al Sistema General de Seguridad Social en Salud, inspeccionando el cumplimiento de las normatividades y de los estándares de calidad en cada una de las instituciones prestadoras de salud para la atención humanizada. Sin embargo, se siguen presentando las inconformidades por parte de los usuarios en manifiesto a la vulneración de sus derechos y la negligencia en la inoportuna atención en los servicios, con negativas en los ordenamientos a procedimientos médicos y retrasos en las atenciones médicas. Finalmente (Yépez Et ál., 2018) manifiesta que el "incumplimiento de las obligaciones por parte de las EPS, negación de servicios y exceso de trámites como barrera de acceso para exámenes y servicios médicos especializados, destacándose las limitaciones de acceso para las poblaciones rurales, precariedad de la infraestructura de las instituciones de salud y limitaciones en la disponibilidad de los profesionales." (2018, p 99)

\section{Articulación de la Bioética con el derecho}

La relación de la bioética y el derecho viene dándose desde hace varios años, donde la 
perspectiva de cada uno de ellos se unifica hacia un mismo objetivo, que es el cuidado y la protección de los derechos fundamentales como la vida, la salud y la dignidad humana desde la ciencia médica y el ejercicio jurídico; pero en Colombia el sistema de salud manejado por el monopolio de las EPS se ve ampliamente vulnerados los derechos fundamentales de los usuarios mayormente cotizantes del régimen contributivo y que actualmente acuden a las instancias de la presentación de acciones de tutela para que sus derechos no sean violados.

Si bien es cierto Milton Rojas nos expresa que los principios que enuncia una Constitución Política de un país representan el mínimo de acuerdo de una sociedad, con respecto a unos derechos y valores inalienables, todo ellos derivados de la Dignidad de la Persona, proponiendo tanto su promoción como su protección. (2006, p 69-70).

Partiendo de esa idea, lo que pretende el hoy llamado Bioderecho es la unanimidad de una atención digna, oportuna y de calidad a los usuarios de las entidades que prestan servicios de salud en el país, por lo cual la Corte Constitucional en virtud de proteger tales derechos emite la sentencia C-313/14 "Mediante oficio de junio 26 de 2013 el Presidente del Senado de la República remitió a esta Corporación el expediente del proyecto de Ley Estatutaria 209 de 2013 Senado y 267 de 2013 Cámara "por medio de la cual se regula el derecho fundamental a la Salud y se dictan otras disposiciones" Corte adelante el estudio oficioso preceptuado por el artículo 241-8 de la Constitución Política".

Roberto Andorno expone "que las actividades biomédicas están en relación directa con las prerrogativas más fundamentales de la persona humana, tales como el derecho a la vida, a la salud y a la integridad física y psíquica. Es lógico, entonces, que el principio de dignidad humana, que es la fuente de la que emergen todos los derechos, se invoque como una justificación última de toda regulación normativa en la materia".

Es preciso enunciar que el principal derecho que vulneran las EPS, IPS y clínicas del país es la dignidad humana de la cual emergen la vida, la salud y que es la principal fuente por la cual acuden al derecho para su respeto y debida sanción, hoy día encontramos que la Corte constitucional dicta sentencias tales como la T-136/04, T-386/18, T-171/18, por medio de las cuales los hechos presentados se basan en la violación o vulneración de sus derechos o de familiares como la dignidad humana, la salud, la vida y en busca o solicitando la protección de los mismos en condiciones dignas de atención por parte de las EPS, además, cabe mencionar que solicitan dentro de sus pretensiones la atención oportuna e inmediata por razones necesarias en condiciones de salud crónicas, donde la calidad de vida se ve tan afectada que incapacita sus funciones tanto físicas como mentales, ocasionando estados de complicaciones y que conllevan al deterioro rápido e inminente de la salud de los usuarios, casos puntuales como los pacientes que han sido diagnosticados con cáncer, muy específicamente viven el día a día de una lucha con su enfermedad y contra su EPS, donde por órdenes medicas deben ser sometidos a tratamientos largos y de alto costo, que deben procurar ser realizados en el menor tiempo posible, las EPS retrasan la expedición de tales órdenes, no cuentan con una red externa de clínicas o entidades especializadas para las debidas atenciones de procedimiento, exámenes y tratamientos, la negación de autorizaciones a medicamentos farmacológicos de alto costo, cobijándose a que solo se les darán los que estén cubiertos por el POS, por tal motivo es aquí donde el derecho entra hacer parte de la triada de la vida y de la mano de bioética a buscar soluciones que permitan que se brinden una mejor atención en salud y de manera oportuna, logrando disminuir los impactos en la salud de los usuarios.

\section{Dignidad Humana, Bioética y Bioderecho}

En relación con la dignidad humana partimos de un mismo objetivo que nos lleva a denotar la importancia de esta, como un derecho inalienable que debe ser protegido desde el ámbito de la ética médica y el campo de 
jurídico y de la cual emanan la esencia del ser humano. Teniendo en cuenta que la dignidad humana anteriormente no era reconocida dentro del marco de una constitución, esta pasa a considerarse derecho fundamental posteriormente a mediados del Siglo XX con la creación de la Carta de las Naciones Unidad (1945) y con la Declaración de los Derechos del Hombre y del Ciudadano (1948), posteriormente consagrada en el Art 1 de la c. (Aparisi., 2013).

Cabe señalar que, para Angela Aparisi, en la actualidad, la dignidad humana se configura como una especie de "conciencia jurídica" global. Incluso, podría sostenerse que, de algún modo, este principio vendría a ocupar el papel que, tradicionalmente, ha desempeñado el Derecho natural, entendido como raíz ontológica y fundamento último del Derecho. (2013., p 204)

Para Miguel Kottow la idea de dignidad humana es la articulación conceptual que conecta la moralidad de igual respeto para todo individuo, con la ley positiva y la legislación democrática de tal modo que su interacción podría dar lugar a un orden político fundamentado en derechos humanos. (2016., p 327)

Hay que mencionar, además, que la Bioética ha surgido con una fuerte intención de tender puentes no sólo entre la ciencia y la ética, sino también entre las diversas mentalidades sociales y culturales, y para ello es imprescindible que no se quede exclusivamente en un ejercicio de toma de decisiones, o en la implementación de modelos éticos que ayuden en las decisiones clínicas para la atención en salud, si no, para una atención donde se brinde calidad y respeto por la dignidad humana de todos aquellos usuarios que soliciten los servicios en salud, tratando de promover dentro del personal sanitario una principal y adecuada relación médico - paciente e instituciones prestadoras de salud - paciente que pretenda el respeto por la dignidad humana, la vida y salud escalando a la mejora continua de las condiciones sanitarias del país. (León., 2007).
En Colombia se puede afirmar que es un Estado social de derecho, organizado en forma de República unitaria, descentralizada, con autonomía de sus entidades territoriales, democrática, participativa $\mathrm{y}$ pluralista, fundada en el respeto de la dignidad humana, en el trabajo y la solidaridad de las personas que la integran y en la prevalencia del interés general. (Quintero-Lobo, 2018)

Así mismo, Milton Rojas asemeja que los artículos 1 y 5 de la Constitución Política garantizan en última instancia el mismo valor (aunque llegan por vías diferentes, el primero vela por el respecto a la Dignidad de la Persona Humana, y el segundo al reconocimiento y primacía de los derechos inalienables del Hombre), el de la protección de la persona en su dignidad. En este punto, la Constitución se encuentra con la totalidad de los Principios Bioéticos, pues todos estos tienden a garantizar el trato digno de la persona. (2006., p 73).

\section{Conclusiones}

En conclusión, la importancia de los principios bioéticos y su articulación con el derecho tiene objetivos similares ya que los dos buscan poner al hombre en el primer plano de priorización para el respeto de sus derechos durante la atención humanizada en las entidades prestadoras de salud, esto se hace necesario su inclusión en el caso del sistema de salud en Colombia, por tal motivo este tipo de análisis puede ser útil para redireccionar su actuar. Asimismo, puede servir para garantizar una calidad en la prestación de los servicios asistenciales de la salud en Colombia.

Finalmente, la existencia de tutelas cuyas sentencias buscan obligar a las entidades de la salud a la restitución de los derechos fundamentales de los usuarios, pone aún más de manifiesto la necesidad de la existencia de un cuerpo de doctrinas que definan los criterios que hagan más humana la prestación de los servicios asistenciales de la salud, por último, se puede decir que es el momento en que el derecho entra hacer parte de la triada, en la defensa de todos aquellos ciudadanos que 
buscan la protección de sus derechos fundamentales, que dentro del marco legal, estos sean vulnerados al momento de la atención en salud.

\section{Referencias Bibliográficas}

León, F. (1997). Dignidad Humana, Libertada y Bioética. Grupo De Investigación En Bioética De Galicia.

Mendoza, A. (2017). La relación médico paciente: consideraciones bioéticas. Simposio: Bioética y Atención de la Salud Sexual y Reproductiva. RevPeru Ginecología y Obstetricia.

Molina, N. (2016). Formación bioética en ciencias de la salud. CiencTecnol Salud Vis Ocul. 14(1):117-32.

Restrepo, J. (2007). La calidad en la prestación de los servicios de salud: un imperativo por lograr. Rev. Fac. Nac. Salud Pública, (25(1):78-81).

Yepez, M., Ricaurte, M., \& Jurado, D. (2018). Calidad percibida de la atención en salud en una red pública del municipio de Pasto, Colombia. Universidad Y Salud, 20(2):97110.

Rojas, M. (2006). Los Principios Bioéticos en los Principios Constitucionales. Opinión Jurídica, (Volumen 5, No. 9)

Corte Constitucional (2014). Colección De Jurisprudencia Colombiana. Recuperado de $<\underline{\text { https://xperta.legis.co }}>$

Andorno, R. (s.f). El Principio de Dignidad Humana en el Bioderecho Internacional. Recuperado de ppct.caicyt.gov.ar\&gt;index.php\&gt;bcae em\&gt;article\&gt;download.

Corte Constitucional, Sala Tercera de Revisión. (19 de febrero de 2004) Sentencia T839394. [MP Manuel Cepeda].

Corte Constitucional, Sala Sexta de revisión. (21 de septiembre de 2018) Sentencia T6.757.944. [MP Gloria Ortiz].

Corte Constitucional, Sala Séptima de Revisión. (7 de mayo de 2018) Sentencia T6.406.033. [MP Cristina Pardo].

Aparisi, A. (2013). El Principio de la Dignidad Humana como Fundamento de un Bioderecho Global. Cuadernos de Bioética, XXIV (2),201-221.

Kottow, M. (2016). Bioética y Derechos (s): Algunos Alcances. Derecho \& Sociedad, $N^{\circ}$ 47 / pp. 321-332.

León, F. (2007). Dignidad Humana y Derechos Humanos en Bioética. Biomedicina Vol. (3), $\mathrm{N}^{\circ} 1,71-81$.

U. Quintero-Lobo, D.P Beltrán-Quintero y M.A Afanador (2019). El control social a lo público educa, mejora nuestra calidad de vida, y es responsabilidad de todos. Sostenibilidad, Tecnología y Humanismo, vol. 10, no. 1 